This document is the accepted manuscript version of the following article:

Ebi, D., Denisov, A., Bonciolini, G., Boujo, E., \& Noiray, N. (2018). Flame dynamics intermittency in the Bistable Region Near a Subcritical Hopf Bifurcation. Journal of Engineering for Gas Turbines and Power, 140(6), 061504 (6 pp.). https://doi.org/10.1115/1.4038326

\title{
FLAME DYNAMICS INTERMITTENCY IN THE BI-STABLE REGION NEAR A SUBCRITICAL HOPF BIFURCATION
}

\author{
D. Ebi \\ Laboratory for Thermal Processes \\ and Combustion \\ Paul Scherrer Institute \\ 5232 Villigen \\ Switzerland \\ Email: dominik.ebi@psi.ch
}

\author{
A. Denisov \\ Institute of Thermal and Fluid Eng. \\ School of Engineering \\ Hochschule für Technik FHNW \\ 5210 Windisch \\ Switzerland \\ Email: alexey.denisov@fhnw.ch
}

\author{
G. Bonciolini, E. Boujo, N. Noiray* \\ CAPS Laboratory \\ Mechanical and Process Eng. Dept. \\ ETH Zürich \\ 8092 Zürich \\ Switzerland \\ Email: noirayn@ethz.ch
}

We report experimental evidence of thermoacoustic bistability in a lab-scale turbulent combustor over a welldefined range of fuel-air equivalence ratios. Pressure oscillations are characterized by an intermittent behavior with "bursts", i.e. sudden jumps between low and high amplitudes occurring at random time instants. The corresponding probability density functions of the acoustic pressure signal show clearly separated maxima when the burner is operated in the bi-stable region. The gain and phase between acoustic pressure and heat release rate fluctuations are evaluated at the modal frequency from simultaneously recorded flame chemiluminescence and acoustic pressure. The representation of the corresponding statistics is new and particularly informative. It shows that the system is characterized, in average, by a nearly constant gain and by a drift of the phase as function of the oscillation amplitude. This finding may suggest that the bi-stability does not result from an amplitudedependent balance between flame gain and acoustic damping, but rather from the non-constant phase difference between the acoustic pressure and the coherent fluctuations of heat release rate.

\section{Introduction}

Large-amplitude oscillations in combustors such as gas turbines and aeroengines are a major threat for the integrity of mechanical components [1]. This kind of oscillations

\footnotetext{
*Address all correspondence to this author.
}

is typically associated with one or several thermoacoustic modes becoming unstable due to the constructive interaction between (i) heat release rate fluctuations $q$ from the flame that responds to acoustic perturbations $p$, and (ii) pressure fluctuations $p$ in the combustor induced by heat release rate fluctuations $q$ that act as an acoustic source.

In general, a thermoacoustic mode becomes linearly unstable when one of the operating conditions (bifurcation parameter $v$, such as equivalence ratio or temperature) exceeds a threshold value (critical value $v_{c}$ ). The system then undergoes a Hopf bifurcation that leads to a limit cycle through the breaking of temporal invariance, as sketched in Fig. 1. In the linearly stable regime $\mathrm{v}<\mathrm{v}_{c}$, coherent perturbations are attenuated and the system is brought back toward the equilibrium state, which is characterized by low-amplitude turbulence-driven thermoacoustic oscillations with $|p| \rightarrow 0$. In the linearly unstable regime $v>v_{c}$, this equilibrium state becomes linearly unstable and small perturbations exponentially grow, giving oscillations of finite amplitude $|p|>0$.

The above picture is purely linear: the linear stability of the system, i.e. the fate of small perturbations, can be deduced from the eigenvalues of the linearized governing equations. What actually happens to non-small perturbations depends on nonlinear effects. In supercritical Hopf bifurcations (left panel of Fig. 1), the dominant nonlinearities have a stabilizing effect that determines the oscillation amplitude $|p|$. In this case, $|p|$ increases continuously from 0 at the critical condition $v=v_{c}$ and instability is therefore detected fairly easily in practice, provided $v$ is varied slowly enough. In sub- 

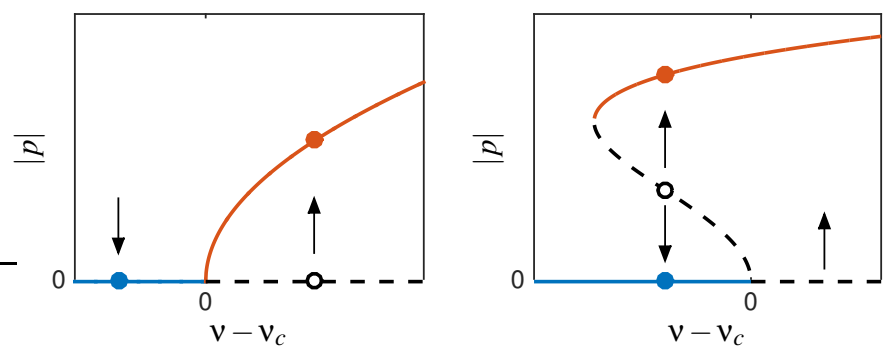

Fig. 1. Sketch of supercritical (left) and subcritical (right) Hopf bifurcations in a purely deterministic situation (without noise). Solid lines: stable fixed point $(|p|=0)$ and stable limit cycle $(|p|>0)$; dashed lines: unstable equilibrium states. Arrows illustrate how stable (resp. unstable) states are attractive (resp. repulsive).

critical Hopf bifurcations (right panel of Fig. 1), the nonlinearities triggered at low amplitude have a destabilizing effect such that the amplitude saturates at a non-vanishing value only when higher-order stabilizing nonlinearities come into play. This results in a bi-stable region, i.e. an interval of the bifurcation parameter where both low- and high-amplitude states are stable. In this region, the presence of noise allows the system to jump back and forth from one state to the other, leading to bimodal probability density functions of the amplitude [2] and to intermittency [3]. Subcritical bifurcations are particularly challenging in practical applications since the system can jump very suddenly from a low-amplitude state to a large-amplitude state, not only when $v$ exceeds $v_{c}$, but also when $v<v_{c}$ if noise is strong enough to bring the system out of the basin of attraction of the low-amplitude state. This situation, when the system is linearly stable but nonlinearly unstable, is also known as "triggering" for rocket combustion instabilities [4]. See also [5] for an example of subcritical bifurcation in industrial gas turbines.

As mentioned earlier, unsteady heat release rate $q$ acts as an acoustic forcing term in the wave equation. The famous Rayleigh criterion $[1,6]$ states that this forcing term is a local source of acoustic energy if the time-averaged product of pressure fluctuations and heat release rate fluctuations is positive over an acoustic period $T$,

$$
\int_{T} p q \mathrm{~d} t>0
$$

For harmonic oscillations, this is verified when the relative phase $\theta$ between $p$ and $q$ satisfies $0^{\circ}<|\theta|<90^{\circ}$, with a maximum constructive interaction when they are in phase with $\theta=0$.

Conversely, the forcing term is a sink of acoustic energy if $\int_{T} p q \mathrm{~d} t<0$, i.e. for time-harmonic fields when $90^{\circ}<|\theta|<$ $180^{\circ}$, with a maximum destructive interference if $p$ and $q$ are out of phase [7]. This criterion applies as well to heat release distributed over a volume $V$ by considering the spatially integrated version of Eq. (1).

It should be noted that the Rayleigh criterion is neither necessary nor sufficient for the system to become linearly unstable. Indeed, even in the absence of acoustic fluctuations, energy can be produced by in-phase fluctuations of entropy (or temperature) and heat release rate $[8,9]$; in this study we focus on acoustic fluctuations and do not consider entropy. In addition, dissipation effects are present in the volume and at the boundaries (acoustic boundary layers, dissipative vortex sound interactions, radiation losses), such that an instability is not triggered when the energy production $\mathcal{P}$ is positive, but only when it overcomes dissipation $\mathcal{D}$,

$$
\mathcal{P}(|p|)=\int_{V} \int_{T} p(t) q(t ;|p|) \mathrm{d} t \mathrm{~d} V>\mathcal{D}(|p|) .
$$

Here we have written explicitly the dependence on the oscillation amplitude in order to emphasize the nonlinear character of the problem. Although bi-stability is often explained as a result of a nonlinearity in the gain $G$ of the coherent heat release rate response to acoustic perturbations $q(|p|)=G(|p|) \cos (\omega t+\theta(|p|))[10-13]$, it is important to note that it can also be induced by a nonlinearity in the phase $\theta[14,15]$.

In this paper, we report experimental evidence of intermittency in a turbulent combustor, with a detailed analysis of chemiluminescence and acoustic pressure measurements, which may suggest that bi-stability is caused by phase nonlinearity rather than gain nonlinearity.

\section{EXPERIMENTAL SETUP}

The measurements were performed in an opticallyaccessible swirl combustor operated at atmospheric pressure [16]. The schematic arrangement of the test rig is shown in Fig. 2. Methane was mixed with preheated air upstream of a flow straightener (not shown here) such that equivalence ratio fluctuations do not participate in the observed combustion instabilities. Swirl is generated by an eight-bladed axial swirler, which has a calculated swirl number of 0.6. The annular mixing section has an inner diameter of $19 \mathrm{~mm}$ (centerbody diameter) and an outer diameter of $41 \mathrm{~mm}$.

Acoustic pressures were recorded upstream of the premix section and in the combustion chamber by four calibrated water-cooled microphones (Brüel\&Kjær, Type 4939). The microphone locations are indicated in Fig. 2. The spatially integrated $\mathrm{OH}^{*}$ chemiluminescence intensity $I_{\mathrm{OH}^{*}}$ was recorded with a photomultiplier equipped with a $310 \mathrm{~nm}$ band-pass filter. The acoustic and integrated $\mathrm{OH}^{*}$ signals were acquired at $10 \mathrm{kHz}$.

The chemiluminescence was recorded simultaneously with a LaVision HSS X camera coupled to a HS-IRO image intensifier at $1 \mathrm{kHz}$. The selective detection of $\mathrm{OH}^{*}$ chemiluminescence was achieved by using a UV-optimized lens

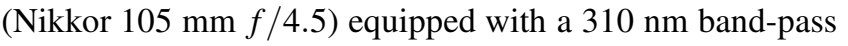
filter. The acoustic data acquisition and chemiluminescence imaging were synchronized in time by a common clock.

\section{BISTABILITY}

The atmospheric pressure experiments were conducted with a combustor inlet velocity of $10 \mathrm{~m} / \mathrm{s}$ and an inlet temperature of $300{ }^{\circ} \mathrm{C}$. The thermal power was about $12 \mathrm{~kW}$. 


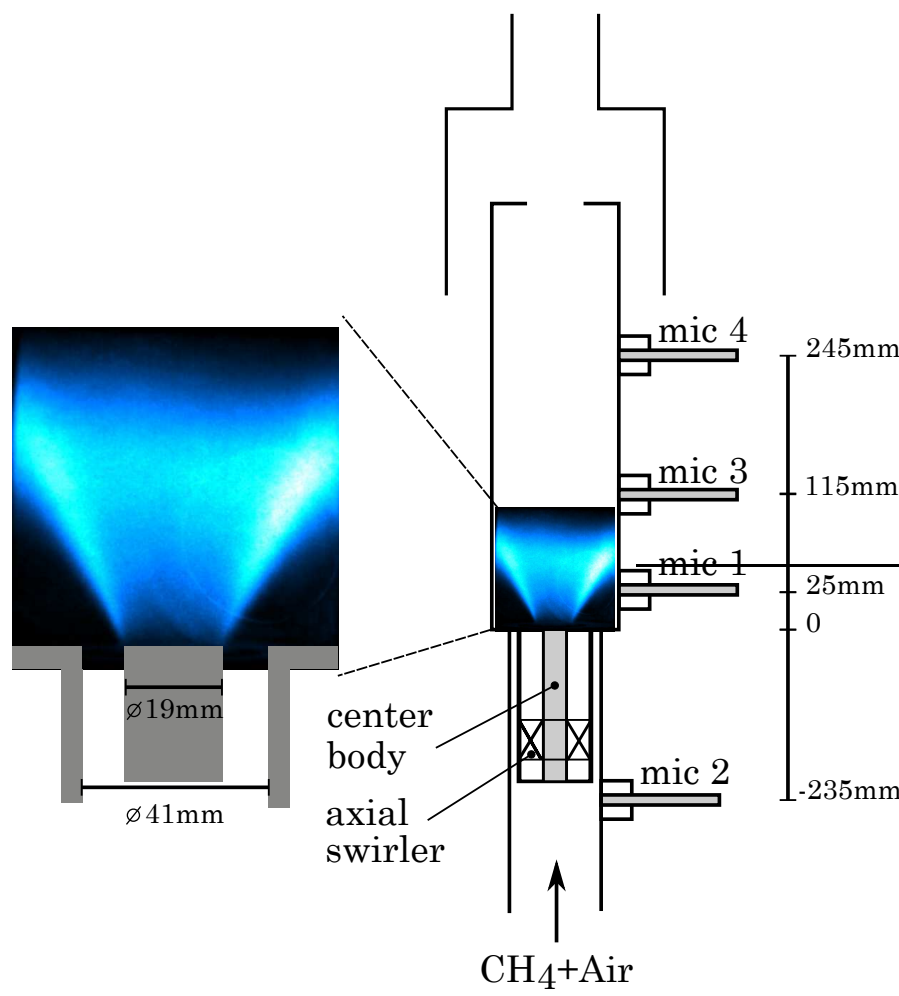

Fig. 2. Schematic of the swirl combustor test rig including microphone locations relative to the burner inlet.

The equivalence ratio $\phi$ of the $\mathrm{CH}_{4}$-air flame was varied to identify a bi-stable regime. The power spectral densities $S_{p p}$ at five different equivalence ratios $\phi$ are shown in Fig. 3b. At $\phi=0.635$ the combustor features a thermoacoustic instability with a center frequency of $150 \mathrm{~Hz}$. A decrease in equivalence ratio leads to a slight shift to successively lower frequencies. At $\phi=0.580$ the combustor is quiet and the frequency associated with the mode of the dominant instability at higher equivalence ratios has decreased to $142 \mathrm{~Hz}$.

Three cycles of (bandpass-filtered) acoustic pressure during high-amplitude oscillations are shown in Fig. 3a for each of the four microphones. Pressure signals from microphones 1, 3 and 4, all located downstream of the burner inlet, are approximately in phase indicating that the investigated eigenmode is established in the entire combustion chamber. Pressure measurements obtained with microphone 3 are used for the analysis in the remainder of the present work.

Time traces of acoustic pressure at each of the five equivalence ratios are shown in Fig. 4. The corresponding probability density functions (PDFs) were constructed from $180 \mathrm{~s}$ of data. The presented time traces (only $50 \mathrm{~s}$ out of the $180 \mathrm{~s}$ are shown for better visibility) are the band-pass filtered pressure signals of microphone 3 located in the combustion chamber (Fig. 2). A rectangular band-pass filter with a sharp lower and upper cut-off at 138 and $160 \mathrm{~Hz}$, respectively, was chosen. The filter width is indicated in Fig. $3 b$ by the dashed lines.

The quiet operating condition (blue, $\phi=0.580$ ), corresponding to a linearly stable system, is characterized by a

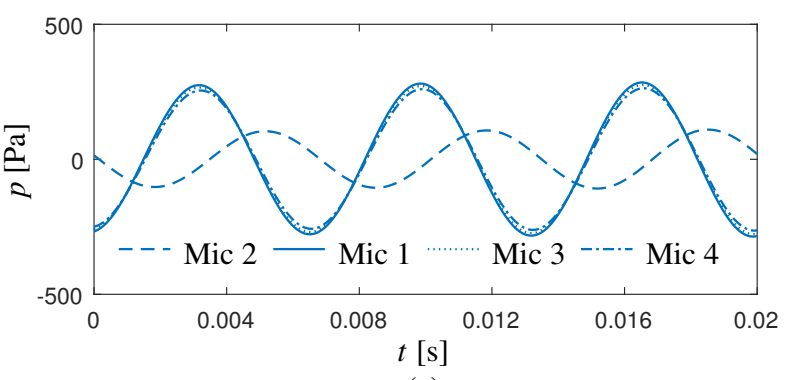

(a)

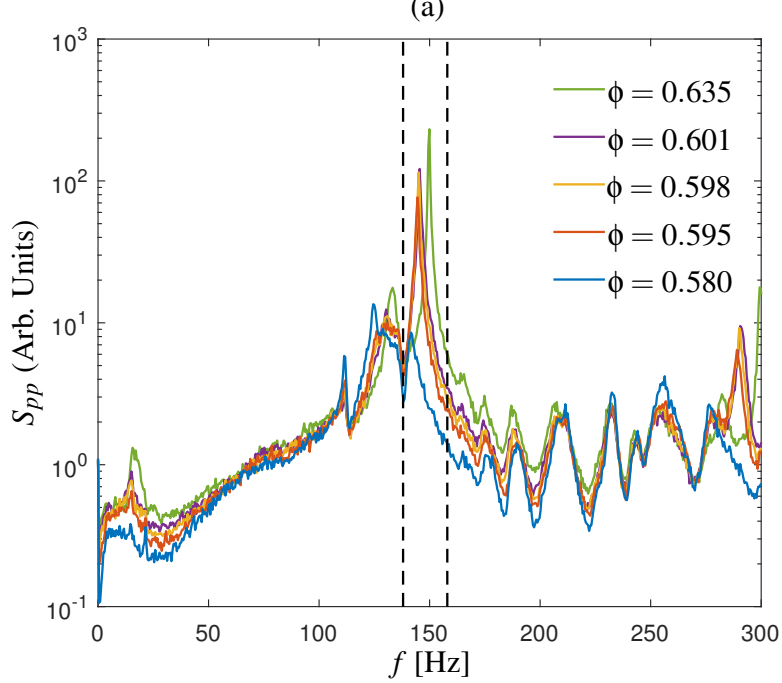

(b)

Fig. 3. (a) Three cycles of band-pass filtered high-amplitude oscillations of all four microphones. (b) Power spectral density of the acoustic pressure recorded with microphone 3 for different equivalence ratios. The focus of this study is the dominant mode at $f \simeq 150 \mathrm{~Hz}$.

Gaussian-like PDF. Beyond a lower bound in equivalence ratio, a bi-stable region exists, which covers a range of about 0.015 in $\phi$ for the investigated swirl combustor and operating conditions. This bi-stable region is characterized by random bursts of high-amplitude oscillations, which are clearly seen in the band-pass filtered time traces at $\phi=0.595, \phi=0.598$ and $\phi=0.601$ (red, yellow and purple, respectively). This intermittency is revealed in the PDF in form of a central peak (corresponding to times when the system is around the stable focus) in addition to the two side peaks (associated with the limit-cycle oscillations).

At $\phi=0.595$, the systems spends about half of the time undergoing high-amplitude oscillations whereas the combustor is still quiet during the other half of the time. As a result, side peaks are starting to form in the PDF in addition to the central peak. At $\phi=0.598$, the system spends more time in a highamplitude oscillatory state. However, the central peak in the PDF still dominates over the side peaks as the acoustic cycle spends more time near zero than at its extrema. At $\phi=0.601$, the system only rarely returns to a low-amplitude state. The central peak in the PDF begins to disappear. Beyond some upper bound in equivalence ratio, the PDF is purely bimodal, signaling that the system undergoes a limit-cycle oscillation 

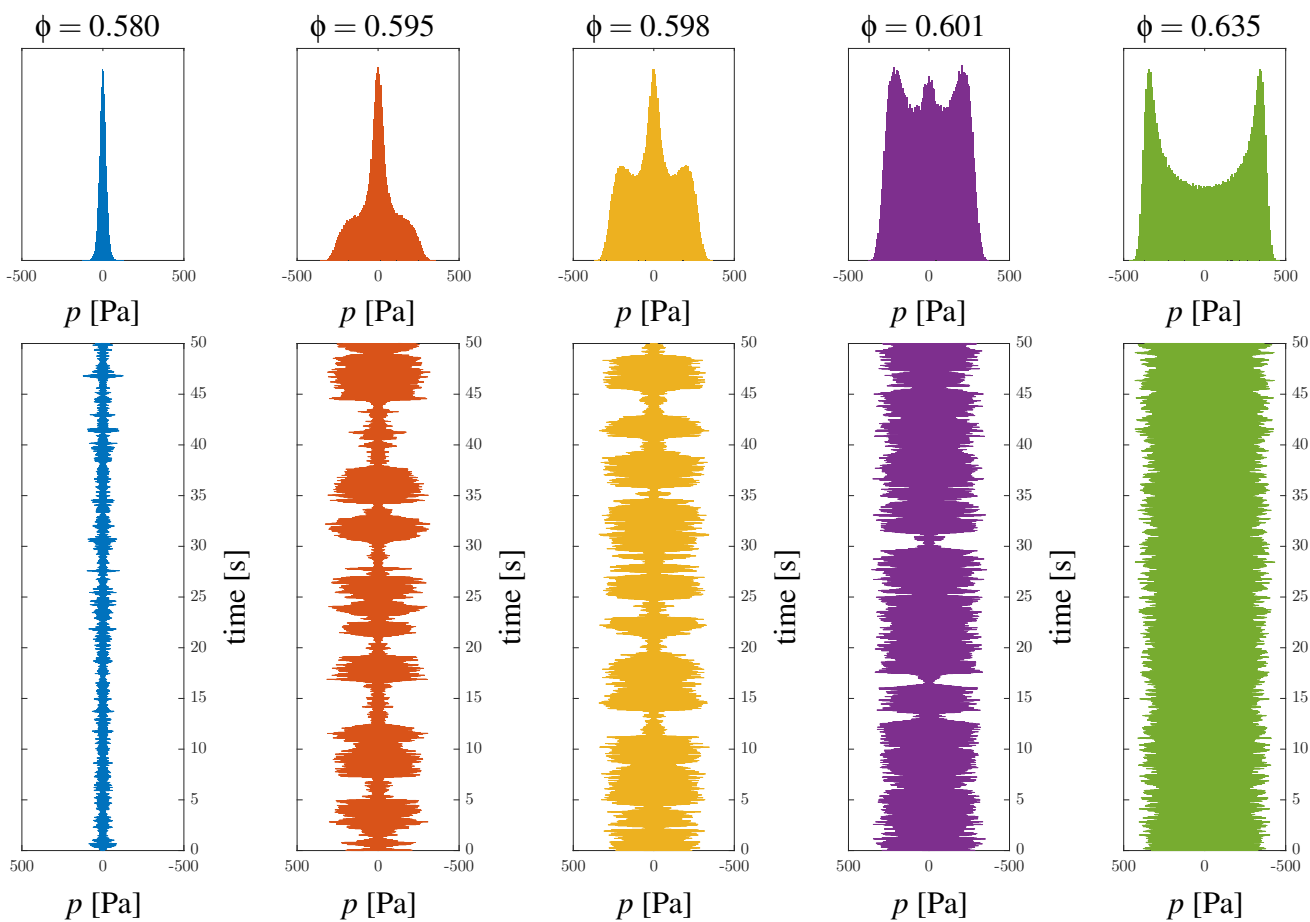

$p[\mathrm{~Pa}]$
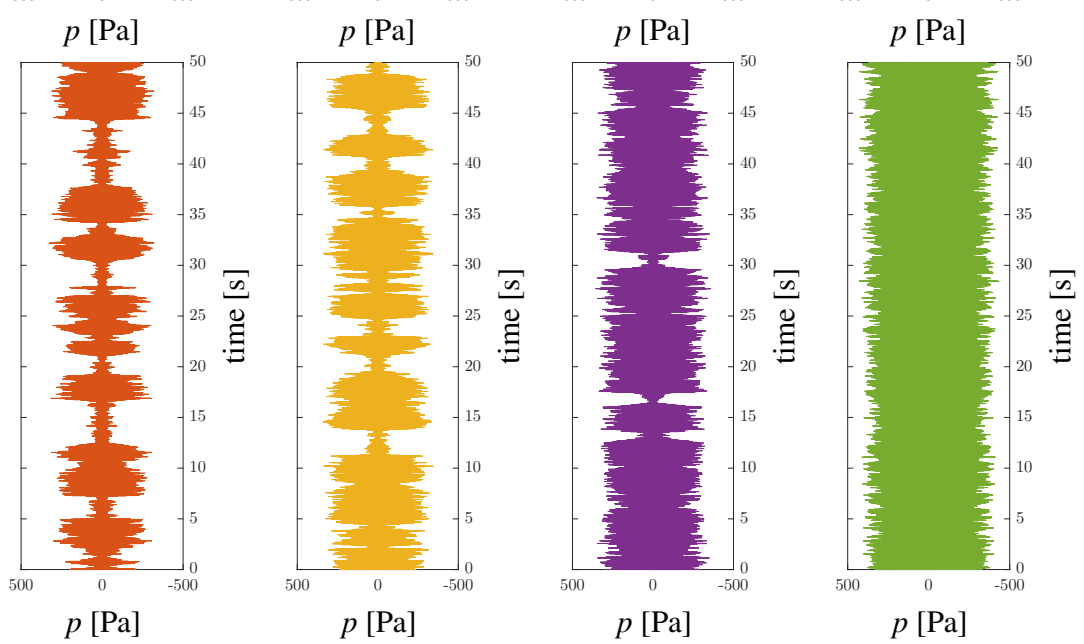

Fig. 4. Acoustic pressure signals (bottom) and probability density functions (top; different $y$ scales) for different equivalence ratios.

at all times as shown here for $\phi=0.635$ (green).

Within the bi-stable operating regime, the change in equivalence ratio required to observe a significant difference in the intermittency was on the order of $0.5 \%$ as indicated in Fig. 4. Note that the absolute accuracies associated with setting the equivalence ratios (combined accuracy of the fuel and air mass flow controller) was about $3 \%$. The relative accuracies (stability) of the mass flow controllers, however, were about one order of magnitude higher. A temporally stable intermittent behavior was therefore achieved (given that the combustion test rig was in thermal equilibrium), which was confirmed by evaluating the PDFs shown in Fig. 4 for subsets of the full time trace.

\section{FLAME SHAPE}

Chemiluminescence images have been acquired in addition to the acoustic data for an operating condition within the bi-stable region $(\phi=0.595)$. The mean flame shape during quiet conditions, which is characterized by a V-shape, is shown in Fig. 2. The gray rectangles indicate the tip of the center body and the burner head, respectively.

The phase-averaged flame shape during high-amplitude oscillations is shown in Fig. 5. The phase angle is based on the pressure trace recorded with microphone 3 . At phase angle $0^{\circ}$, the flame exhibits a kink. With subsequent phase angles the flame becomes more compact and the flame root further distances itself from the centerbody rim. After reaching its most compact shape at phase angle $90^{\circ}$, the flame begins to elongate again starting at $135^{\circ}$. The flame root propagates upstream during this phase. By phase angle $225^{\circ}$ the flame has reached its maximum axial extent and the flame root is attached to the center body. A pronounced kink in the flame front forms again at phase angle $270^{\circ}$, which is subsequently convected downstream.

\section{AMPLITUDE-CONDITIONED STATISTICS}

Different mechanisms can lead to the bi-stability of the combustor, observed in Fig. 4. In [12], the transition from quiet to loud regime is induced by a nonlinearity of the flame response to acoustic perturbations, as shown in the left panel of Fig. 6. Each point of this scatter plot represents one possible state visited by the combustor, in terms of chemiluminescence intensity $I_{\mathrm{OH}^{*}}$ and acoustic amplitude, at a given operating condition. Even if to a higher acoustic perturbation corresponds a higher heat release fluctuation, the underlying trend is non-linear as highlighted by the red dashed line. This means that whenever the state of the system driven by the turbulence-induced combustion noise crosses a certain threshold in acoustic perturbation, a more than proportional increase of heat release rate fluctuation is generated. This promotes the rise of a high-amplitude pressure limit cycle, as it constitutes a source term for acoustic pressure.

In contrast, the combustor and operating condition investigated in the present study reveal a different behavior. As shown in the right panel of Fig. 6, the relation between heat release rate and dynamic pressure fluctuations is approximately linear over the whole range of amplitudes at this equivalence ratio in the bi-stable region $(\phi=0.598)$. In 

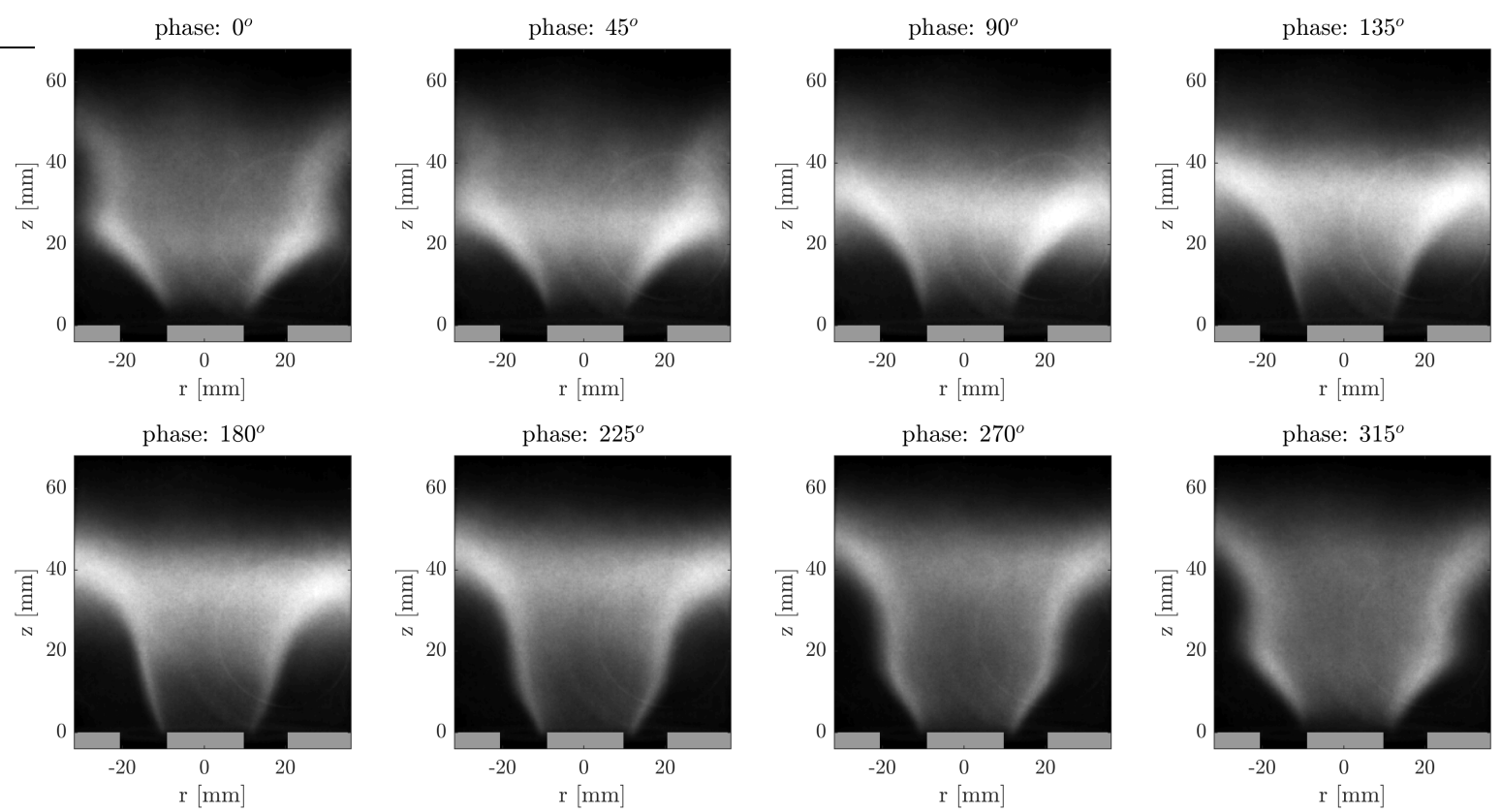

Fig. 5. Line-of-sight $\mathrm{OH}^{*}$ chemiluminescence intensity at different phase angles. High-amplitude regime $(\phi=0.595)$.
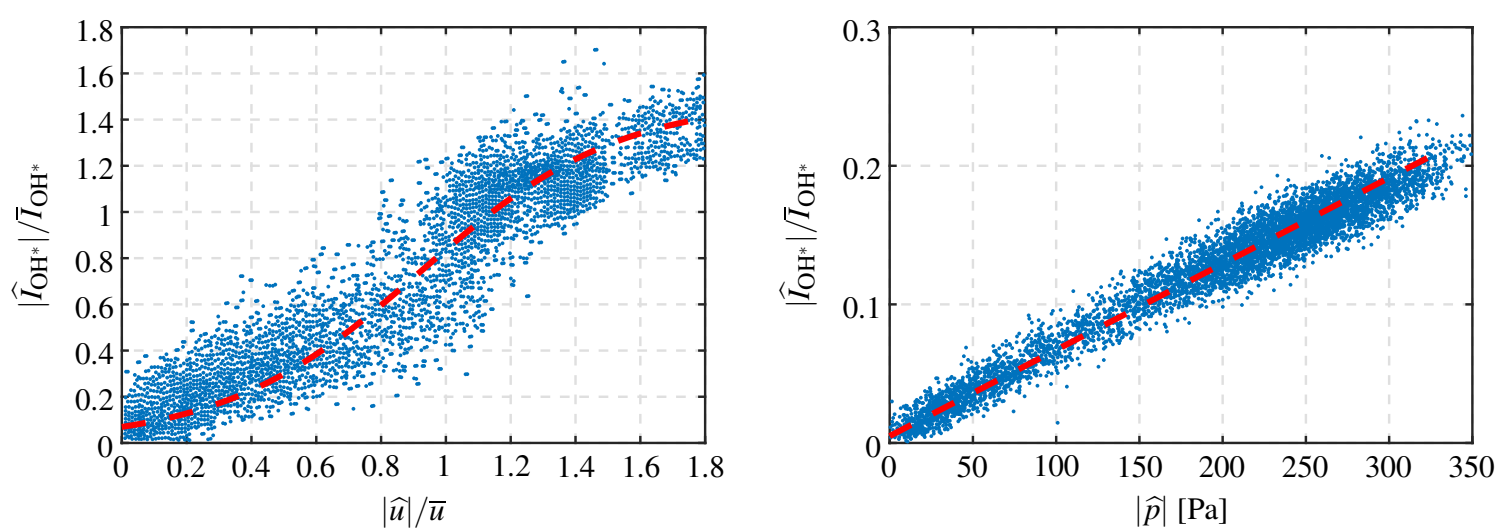

Fig. 6. Normalized flame response: integrated chemiluminescence intensity vs. acoustic oscillation amplitude, for two different combustors. Left: T.U. Berlin combustor (adapted from [12]). Right: PSI combustor (present study) at operating condition $\phi=0.598$.

other words, the gain $G=\left|I_{\mathrm{OH}^{*}}\right| /|p|$ (which is the slope of the dashed red line in the right panel of Fig. 6) is approximately constant. ${ }^{1}$

The joint PDFs $P\left(A_{\mathrm{ac}}, G\right)$ of normalized acoustic amplitude $A_{\text {ac }}$ (envelope of the acoustic pressure signal normalized by the peak pressure amplitude of about $450 \mathrm{~Pa}$ ) and gain $\mathrm{G}$ are shown in the top row of Fig. 7 (the darker the area, the more likely the states). Reading form left to right, i.e. for increasing equivalence ratio, the PDF changes according to the stability property of the system. In the stable case $(\phi=0.580)$,

\footnotetext{
${ }^{1}$ The gain is even slightly decreasing with amplitude as will be highlighted in the top row of Fig. 7. Assuming linear acoustics (which is reasonable for the conditions of the present study), one can conclude that this quasi-linear dependence of $\left|\widehat{I}_{\mathrm{OH}^{*}}\right| / \bar{I}_{\mathrm{OH}^{*}}$ upon $|\widehat{p}|$ means that the gain of the "more conventional" describing function $\left(\left|\widehat{I}_{\mathrm{OH}^{*}}\right| / \bar{I}_{\mathrm{OH}^{*}}\right) /(|\widehat{u}| / \bar{u})$ should also be constant at that operating condition.
}

the system is characterized by low-amplitude oscillations at all times (high values of the PDF are concentrated in the left part of the plot corresponding to small $A_{\text {ac }}$ ).

At $\phi=0.595$ and $\phi=0.598$, the bi-stability appears clearly: the range of amplitudes is broad with two distinct peaks in the marginal PDF $P\left(A_{\mathrm{ac}}\right)$ (middle row), corresponding to low- and high-amplitude oscillations. With a further increase in the equivalence ratio, the system leaves the region of bistability and approaches a stable limit cycle regime, which is fully established at $\phi=0.635$.

In all cases, however, the gain $G$ distribution does not show any strong trend, i.e. the gain is on average approximately constant (even slightly decreasing) across all visited amplitudes. Therefore, a nonlinearity of the gain does not seem to be the governing mechanism that drives the onset of the 

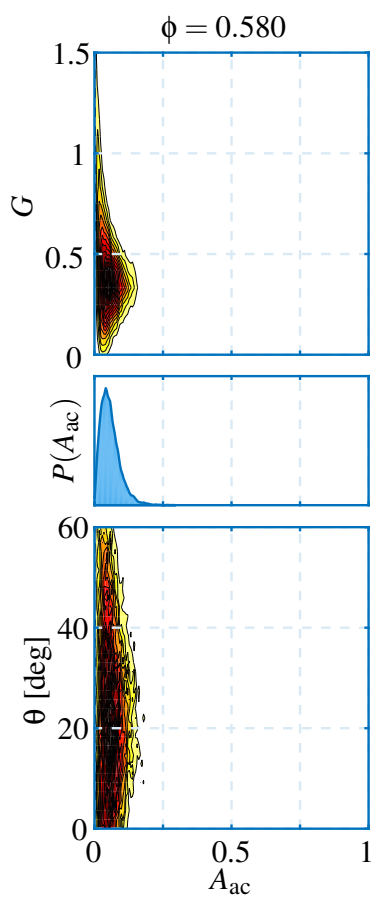

$\phi=0.595$
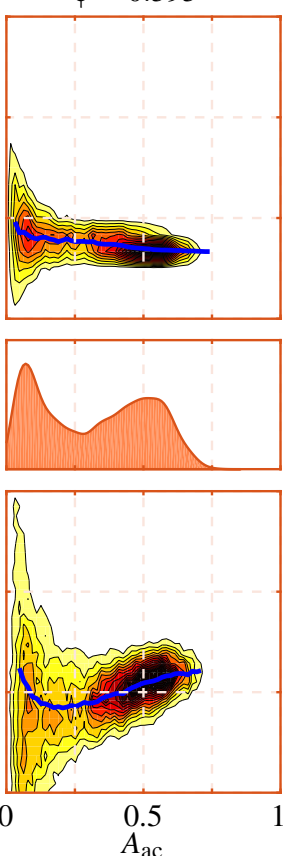

$\phi=0.598$
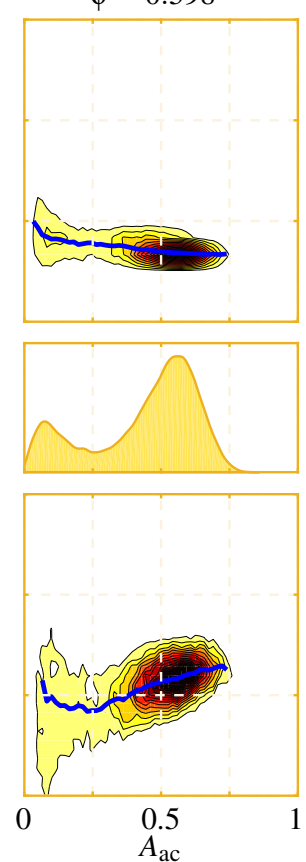

$\phi=0.601$
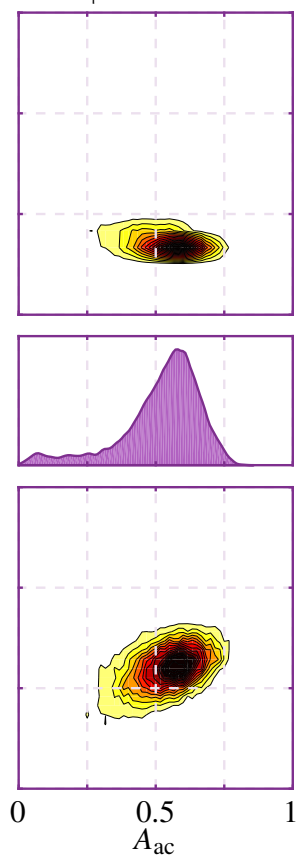

$\phi=0.635$

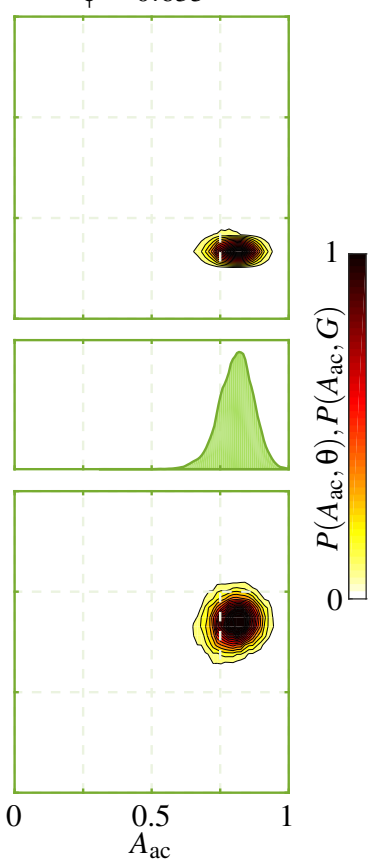

Fig. 7. Joint probability density function of normalized acoustic pressure amplitude $A_{\mathrm{ac}}$ with gain $G=\left|I_{\mathrm{OH}}\right| /|p|$ (top row), and with phase $\theta=L\left(I_{\mathrm{OH}^{*}}, p\right)$ (bottom row), for different equivalence ratios. In the central row, as a reference, the marginal PDF for the acoustic amplitude $P\left(A_{\mathrm{ac}}\right)$.

bi-stability.

The bi-stability might instead be explained via an observed change in the phase between heat release rate and pressure oscillations. Contour maps of the joint probability density function $P\left(A_{\mathrm{ac}}, \theta\right)$ of acoustic amplitude $A_{\mathrm{ac}}$ and phase angle $\theta$ between band-pass filtered chemiluminescence fluctuations and acoustic pressure recorded with microphone 3 are shown in the bottom row of Fig. 7. During low-amplitude oscillations $(\phi=0.580)$, the system covers a rather wide range in phase with a most probable $\theta$ of about $23^{\circ}$. In the bistable operating regime $(\phi=0.595$ and $\phi=0.598)$, the joint PDFs reveal that as the system switches from low- to highamplitude oscillations, the phase angle first decreases and subsequently increases again with an increase in acoustic pressure. This dip in $\theta$ is highlighted by the blue line corresponding to the mean phase at each $A_{\mathrm{ac}}$.

The phase angle plays a fundamental role in the establishment of a thermoacoustic instability, as indicated by the instability criterion Eq. (2). The interaction is constructive if heat is provided at the moment of highest compression, i.e. if $q$ and $p$ are in phase. Therefore, the stability of the thermoacoustic system is affected as much by the magnitudes of $q$ and $p$ as by their relative phase. One can for instance refer to [17] where the evolution of this phase difference was investigated for a monotonic change of the equivalence ratio. Note that in contrast to [17], we focus here on stationary operating conditions, which allows us to extract the amplitudedependent phase difference.

The initial decrease in $\theta$ as $A_{\mathrm{ac}}$ increases indicates that the acoustic energy production increases, which presumably triggers the system to switch to high-amplitude oscillations.
As the pressure amplitude grows beyond some threshold (here: $A_{\text {ac }} \approx 0.2$ ), the phase angle $\theta$ increases again indicating a decrease in acoustic energy production required to establish a new equilibrium between acoustic forcing and damping during limit cycle oscillations.

In summary, the investigated random bursts (intermittent switching between quiet and loud conditions) triggered by stochastic forcing in this highly turbulent configuration are associated with a nearly constant gain but a change in phase. The phenomenon of subcritical bifurcations induced by an amplitude-dependent phase-lag between heat release rate and acoustic pressure has already been observed in previous publications dealing with laminar premixed flames $[15,18]$. It is here encountered in a swirled turbulent flame and the critical role of the non-constant phase as function of the amplitude is drawn from the statistics presented in Fig. 7, which is, to the authors' knowledge, a new way of presenting data that are customarily acquired for thermoacoustic investigations.

\section{CONCLUSION}

In this study, the thermoacoustic dynamics of a turbulent combustor operated at atmospheric pressure has been investigated at stationary conditions, which are characterized by a bi-stable regime. The pressure traces are processed using acoustic amplitude conditioning. This processing method serves to highlight the differences between the quiet and high amplitude oscillating states which are visited by the thermoacoustic system at a given operating condition in the bi-stable range. The main outcome of this study is the statistical representation of the amplitude ratio and phase difference be- 
tween acoustic pressure and chemiluminescence oscillations conditioned on the acoustic level. It suggests that, in this particular configuration, the presence of the subcritical bifurcation and its associated bi-stable region might result from an amplitude-dependent phase difference and, therefore, an amplitude-dependent Rayleigh integral.

\section{Acknowledgements}

E. B. and N. N. acknowledge support by Repower and the ETH Zurich Foundation. G. B. and N. N. acknowledge support by the Swiss National Science Foundation (Grant 160579). Jonas Moeck is also acknowledged for the interesting discussion about the draft paper.

\section{References}

[1] Culick, F. E. C., 2006. Unsteady motions in combustion chambers for propulsion systems. RTO AGARDograph AG-AVT-039, RTO/NATO.

[2] Burnley, V. S., and C. Culick, F. E., 2000. "Influence of random excitations on acoustic instabilities in combustion chambers". AIAA Journal, 38(8), pp. 1403-1410.

[3] Waugh, I. C., and Juniper, M. P., 2011. "Triggering in a thermoacoustic system with stochastic noise". International Journal of Spray and Combustion Dynamics, 3(3), pp. 225-241.

[4] Oefelein, J., and Yang, V., 1993. "Comprehensive review of liquid-propellant combustion instabilities in F1 engines.”. Journal of Propulsion and Power, 9(5), pp. 657-677.

[5] Lepers, J., Krebs, W., Prade, B., Flohr, P., Pollarolo, G., and Ferrante, A., 2005. "Investigation of thermoacoustic stability limits of an annular gas turbine combustor test-rig with and without Helmholtz-resonators". In AMSE Turbo Expo: Power for Land, Sea, and Air, pp. 177-189.

[6] Rayleigh, J., 1896. The Theory of Sound. New York: Macmillan and Co.

[7] Lieuwen, T., 2012. Unsteady Combustor Physics. Cambridge University Press.

[8] Nicoud, F., and Poinsot, T., 2005. "Thermoacoustic instabilities: Should the Rayleigh criterion be extended to include entropy changes?". Combustion and Flame, 142(12), pp. 153 - 159.

[9] Bothien, M., 2008. "Impedance tuning: A method for active control of the acoustic boundary conditions of combustion test rigs". PhD thesis, Technische Universität Berlin.

[10] Balasubramanian, K., and Sujith, R., 2008. "Thermoacoustic instability in a rijke tube: Non-normality and nonlinearity". Physics of Fluids, 20(4), p. 044103.

[11] Preetham, Santosh, H., and Lieuwen, T., 2008. "Dynamics of laminar premixed flames forced by harmonic velocity disturbances". Journal of Propulsion and Power, 24(6), pp. 1390-1402.

[12] Moeck, J. P., Bothien, M. R., Schimek, S., Lacarelle, A., and Paschereit, C. O., 2008. "Subcritical thermoa- coustic instabilities in a premixed combustor". In 14th AIAA/CEAS Aeroacoustics Conference.

[13] Kim, K., and Hochgreb, S., 2012. "Measurements of triggering and transient growth in a model leanpremixed gas turbine combustor". Combustion and Flame, 159(3), pp. 1215-1227.

[14] Bellows, B. D., Neumeier, Y., and Lieuwen, T., 2006. "Forced response of a swirling, premixed flame to flow disturbances". Journal of Propulsion and Power, 22(5), pp. 1075-1084.

[15] Noiray, N., Durox, D., Schuller, T., and Candel, S., 2008. "A unified framework for nonlinear combustion instability analysis based on the flame describing function". Journal of Fluid Mechanics, 615, pp. 139-167.

[16] Hubschmid, W., Denisov, A., and Biagioli, F., 2014. "Acoustic forcing on swirling flow: experiments and simulation”. Experiments in Fluids, 55(9), p. 1808.

[17] Hong, S., Shanbhogue, S. J., Speth, R. L., and Ghoniem, A. F., 2013. "On the phase between pressure and heat release fluctuations for propane/hydrogen flames and its role in mode transitions". Combustion and Flame, 160(12), pp. 2827 - 2842.

[18] Boudy, F., Durox, D., Schuller, T., Jomaas, G., and Candel, S., 2011. "Describing function analysis of limit cycles in a multiple flame combustor". J Eng Gas Turb Power, 133(6), p. 061502. 